expose the surface of the cestode. On the following morning at half-hourly intervals three capsules, each containing I c.c. of the ext. felix mas, are administered. For children I minim is given for each year of life in each dose. Two hours later a second sodium sulphate purge should wash away the tapeworm which has been paralysed by the action of the felicin. Care must be taken to confirm the expulsion of the head. Should the treatment be unsuccessful in removing the head, the same ritual of treatment should be repeated after an interval of ten days.

An even more efficient, but rather more dangerous, method is the use of carbon-tetrachloride. The same ritual as above is followed, except that the fats need not be excluded, and a 3 c.c. capsule is administered in one dose. For children 3 minims are given for each year of life. The toxic effects of this drug may produce in susceptible subjects symptoms of acute liver atrophy -intense jaundice, subcutaneous haemorrhages, and eventually convulsions and death. For this reason it should never be administered to patients who have been addicted to alcohol, or who suffer from cirrhosis of the liver or chronic renal disease. It has been found that by giving large doses of calcium lactate and gluconate, both by mouth and intramuscularly for three days before the treatment, the toxic symptoms are greatly reduced or altogether avoided.

Phenobarbitone and bromide are the limited available drugs during the late irritative phase of the disease.

Surgical intervention is of no avail, as the sites of irritation are always multiple, but decompression may be necessary for the relief of raised intra-cranial pressure.

\title{
REFERENCES
}

BRAILSFORD, J. F., British Journal of Radiology, "Cysticercus cellulosæ-Its radiographic detection in the musculature and central

DIXON, H. B. F., and SMITHERS, D. W., "Epilepsy in Cysticercosis (Tænia Solium). A study in Seventy-one Cases," Quart. Jour. $M e d$ (1934). 3, 603-6r6.

LEUCKART, R., Die Parasiten des Menchen, I, 6r7-7rr.

MACARTHUR, W. P., "Cysticercosis as a cause of Epilepsy in Man," Trans. Roy. Soc. Trop. Med. and Hyg. (1933). 26, 525-528. Mr Costish Army, with Special Reference to the Production of Epilepsy," Ibid. (1934). 27, 343-363. Cases," British Medical Journal (I934). Ir3-14.

\section{SURGERY IN RELATION TO MENINGITIS}

\author{
By HARVEY JACKSON, F.R.C.S.(Eng.) \\ (Hon. Neur. Surg. St. Thomas's Hosp., Regional Consultant, E.M.S., etc.)
}

Exploring the role of surgery in meningitis unfolds an ever-expanding field. How best to peruse the subject is a moot decision, but two main aspects loom to the fore: firstly, the problem of the acute stage of the disease, and secondly the sequelae arising out of incomplete resolution of the inflammatory processes.

\section{Acute Meningitis}

Admittedly in the acute stage of meningococcal meningitis, and other forms of primary meningitis, apart from lumbar puncture or the comparable operations of cisternal or ventricular tapping, surgical intervention assumes but limited application. The position in relationship with possible sequelae, however, is somewhat different, for the formation of scar tissue is apt to induce obliterative changes within the cerebro-spinal fluid pathways, thereby causing obstruction within the subarachnoid spaces of the brain, or the spinal cord.

With secondary varieties of meningitis, such as the septic type, the surgical aspect is paramount, as immediate relief is to be sought in the eradication of the primary focus of infection. The source of infection, nevertheless, is not always so obtrusive, because either the pathway of transmission is not revealed, or lack of co-incident symptoms veils the picture-in other words, a fissured fracture involving the accessory nasal sinuses may not be visualised on the radiograph, or the manifestations of meningitis in a patient with middle ear disease may not be predetermined by the loss of cochlear or vestibular function. The perforating qualities of a particular wound can escape recognition pending the development of meningeal infection; such a lack of realisation may be accounted for out of the limited dimensions of the surface wound, 
or its anatomical dispositions, e.g. wounds through the conjunctival sac. Occasionally the source of intracranial infection is located in scalp infection, emissary venous channels effecting adequate portals of entry.

All cases of suppurative meningitis are not dependent on the spread of infection by continuity of tissue; some indeed are metastic as when a staphylococcal meningitis succeeds a furuncle or infected gnat-bite anywhere on the body surface.

\section{Meningitis due to Contamination.}

The disaster of meningitis arising out of contamination of surgical origin is to be deplored. That such a complication of lumbar puncture should occur reflects on the technique of the procedure, but it may well be the outcome of wrongful application of what is usually a harmless undertaking. Contra-indications to the employment of lumbar puncture are to be found in infected penetrating injuries of the head and in cases of suspected epidural spinal abscess.

\section{Meningitis of Chemical Origin.}

The precipitation of acute meningeal inflammatory reactions by physical or chemical agents is not fully appreciated. A reminder of the irritant properties of disintegrating red blood corpuscles bears citation. Well does one recollect the intense headache, photophobia, nuchal retraction and rigidity, and easily elicited Kernig's sign of a case of subarachnoid haemorrhage, consequent on either a ruptured congenital aneurysm, or a cerebral injury.

Another chemical substance prone to induce meningitis, but of rare incidence, is found in the content of epidermoid tumours. Indeed such is the risk of diffuse chemical meningitis succeeding operative intervention for intradural epidermoids, that the operative mortality for these lesions is distressingly high.

\section{Post-Meningitic Sequelae.}

So much for the acute stage of the disease. What of its sequelae? Resolution of the inflammatory processes as in other parts of the body is rarely complete; in consequence scar tissue is fashioned, so producing compressive or obstructive states. The anatomical disposition of resultant cicatrices may be responsible for loculation of the cerebro-spinal fluid within circumscribed zones, or may block channels such as the iter, or the foramen of Majendie, thereby causing internal hydrocephalus. Obviously varied forms of lesion are possible as the end result of generalised meningitis; amongst these one meets arachnoidal cysts, obstructive or communicating hydrocephalus, and chronic arachnoiditis. The final state may be of localised or generalised distribution.

\section{Post-Meningitic Hydrocephalus}

Post-meningitic hydrocephalus follows on obliterative changes within the normal cerebrospinal fluid pathways, but the site of blockage is not constant. Strictural changes in the iter, fibrotic membranes roofing the foramen of Majendie, arachnoidal adhesions within the hiatus tentorii, and interference with absorptive function of the arachnoidal villi are all possible sources of derangement of the cerebro-spinal fluid circulation. The clinical picture is that of rising intracranial tension depicted in headache, vomiting and papilloedema, but commonly without evidence on which to base the level of obstruction. That such a state is subsequent to acute meningitis reveals its origin, thus differentiating it from other causes of this syndrome (cerebral tumour).

All cases of post-meningitic headache do not find explanation in this manner, for some cases afford little evidence of obstruction. Psychogenic factors play some part in the nonobstructive variety.

Whilst scarring may be diffuse, it is more apt to occur at certain sites, especially in regions where some degree of stasis exists naturally. Thus the subarachnoid spaces traversing the hiatus tentorii appear to be selective, as are the precincts of the basal cistern. In the former situation internal hydrocephalus is the result, but recognition of this from lesions at other levels is only to be determined by employing contrast radiology (ventriculography). 


\section{Chiasmal Arachnoiditis}

Adhesive conditions in the basal cistern are restricted at times to the region of the optic chiasma, wherein strangulation or distortion effects take place. The resultant chiasmal arachnoiditis produces deterioration in the fields of vision, circumferential and/or insular, and diminution of visual acuity together with atrophic changes in the optic discs. . These field changes provide a chiasmal syndrome which together with the history establish the diagnosis. The field defects of arachnoiditis usually differ from those characterising a pituitary tumour in their irregularity. Further points of differentiation are to be found in X-ray appearances and chemical examination of the cerebro-spinal fluid. It is not to be assumed, nevertheless, that chiasmal arachnoiditis arises invariably in such a way, as similar findings may be the outcome of infection in adjacent structures, especially the sphenoidal air sinus.

\section{Spinal Arachnoiditis and Meningitis Serosa Circumscripta}

The spinal canal exhibits similar changes in its contents; the severity of the affliction varying in degree from case to case. For instance, the strangulation effect can be so gross that in association with concurrent vascular disorders a diffuse degenerative softening results, on the other hand the resultant change can be entirely circumscribed and in the form of a single cyst. With a less severe but diffused arachnoidal reaction it is not rare for a somewhat extensive obliterative change to occur, the so-called "chronic spinal arachnoiditis." Thus one may encounter a diffuse condition causing a partial spinal block, or a localised collection of fluid producing a complete block (meningitis serosa circumscripta).

\section{Clinical Indications.}

Apart from the history is there any means of suspecting these various changes? When a patient, in whom a paraplegic state is developing, depicts a sensory disturbance of patch distribution, indicating multiple levels, and the uppermost of those levels fades over. a segment or segments, particularly if there is depression of one or more of the deep reflexes of the lowe limbs, the underlying disease is probably that of chronic arachnoiditis.

Meningitis serosa circumscripta affords a clinical picture of spinal compression consistent with tumour formation.

\section{Pathological Investigations.}

Evidence forthcoming from examination of the cerebro-spinal fluid is not typical. The presence of a complete spinal block of course would be manifest by the usual manometric phenomena with a lack of response on jugular compression, whilst the fluid on analysis would be found to contain a total protein raised to a considerable degree. With cases of spinal arachnoiditis the manometric findings vary: rarely do they indicate a complete block, and frequently the protein content of the fluid is normal.

The presence of a chiasmal syndrome arising out of meningitis would not show any change in the cerebro-spinal fluid, whereas the presence of a pituitary adenoma is associated with a raised total protein content.

The deterioration of a patient following a head injury may be met by lumbar puncture and the presence of a large proportion of blood revealed. It is as well under these circumstances not to accept haemorrhage as conclusive, but to examine the cerebro-spinal fluid for organisms. Some of the most acute cases of infective meningitis develop within a matter of twelve to twentyfour hours of head injury.

\section{Radiographic Examination.}

Plain X-ray affords little aid in the diagnosis of post-meningitic sequelae. On the other hand, contrast radiology is not only helpful, but essential to location of the site of the lesion. For cranial cases without increased intracranial pressure, particularly in confirmation of chiasmal arachnoiditis, encephalography is of use. When increased intracranial pressure is present ventriculography is indicated, for by this method alone can one decide, prior to operation, at 
what level the obstruction is to be found, and so to some extent direct the nature and route of the intervention.

The application of lipiodol myelography to the spinal sequelae affords accurate localisation of the obstruction. With arachnoiditis the lipiodol is held up at multiple levels, and the appearance of the radiograph simulates the gutterings of a candle.

In children with post-meningitic obstruction of long-standing plain X-rays of the skull may show signs of prolonged intracranial pressure, e.g. distension of the sutures, erosion of the posterior clinoid processes, and a hammer-marked vault.

\section{Treatment.}

Prophylaxis is important where this can be applied, hence the avoidance of lumbar puncture when this procedure is contra-indicated. There is one particular case where the principle is well practised, and that is the condition of persistent cerebro-spinal rhinorrhoea-only by closure of the fistula is the inevitable attack of meningitis to be avoided.

During the acute phase of infection the surgeon will rely on chemotherapy to no less a degree than does his medical colleague, and sulphonamides will be applied as indicated in other articles of this series. Whether or not the individual surgeon feels it incumbent that some form of sulphonamide be applied to wounds, a word of caution would not be amiss in stating definitely that sulphathiazole is to be avoided in penetrating wounds of the head. In the opinion of the writer local instillations of sulphanilamide afford little protection in any case, and in no way ameliorate the radical surgical measures otherwise required.

Acute septic meningitis demands immediate eradication of the primary focus of infection wherever that is feasible-e.g. an infected mastoid, an infected frontal sinus, a penetrating injury, or a compound fracture.

The problem of chemical meningitis is related to the causative agent. Blood in the subarachnoid space will require removal by lumbar puncture, when it is causing pain and spasm or other severe reaction. Meningitis due to the intradural release of epidermoid content requires the adoption of a mode of attack whereby the subarachnoid space is sealed off prior to the evacuation of the cyst. itself.

The treatment of the sequelae requires separate consideration, for each is a problem unto

Post-menigitic hydrocephalus necessitates preliminary ventriculography in order that the level of the obstruction may be revealed. The surgical problems attached to the relief of the obstruction are somewhat intricate, but all in common demand the establishment of a free circulation between the ventricular system and the subarachnoid space. Just what type of communication is to be made available is indicated by the level of the obstruction, the several possibilities being: ventriculostomy, division of adhesions over the roof of the fourth ventricle, division of the tentorium. Should these methods fail, or should the fault rest in inadequate absorption of the cerebro-spinal fluid, there remains the possibility of restoring the balance between secretion and absorption by means of suppressing secretion from the choroid plexuses, using deep X-ray therapy: (In the writer's opinion improvement in patients harbouring tumours is at times induced in this way, especially when clinical improvement occurs from teletherapy in the presence of a radio-insensitive neoplasm.)

Chiasmal arachnoiditis is to be met by an exploration at which the optic nerves and the optic chiasma are freed of adhesions, and any collections of fluid dispersed. Although many cases of dramatic restoration of vision are reported, a guarded prognosis in respect thereof is a wise precaution.

Chronic spinal arachnoiditis does not lend itself well to surgical intervention, yet it is by no means uncommon for considerable improvement to follow on exploratory laminectomy.

Meningitis serosa circumscripta, acting as it does as a local compression of the spinal cord, offers the prospect of satisfactory recovery. But it is as well to recollect that this form of lesion is a remnant of a diffuse disease, and in consequence it may be correlated with other changes less responsive to operative relief. 